\title{
Bienestar, Valores y Clima Emocional en Personas Expuestas a Procesos de Violencia Política en Ayacucho, Perú
}

\section{Well-being, Values, and Emotional Climate in People Exposed to Processes of Political Violence in Ayacucho, Peru}

\author{
Henry Guillén y Rosa María Cueto \\ Pontificia Universidad Católica del Perú
}

\begin{abstract}
El periodo de violencia acontecido en el Perú entre los años 1980 y 2000 generó secuelas psicosociales significativas en su población, principalmente en aquella ubicada en la sierra y selva central. Ayacucho, región de la sierra central, es identificada como la más afectada por este proceso. El presente estudio analizó las relaciones entre el bienestar, los valores y el clima emocional en 120 personas que vivieron en Ayacucho durante esos años, seleccionadas por muestreo intencionado. Se obtuvieron correlaciones directas entre valores de motivación humanitaria y de orientación central y social con clima emocional positivo y bienestar subjetivo y social. Por medio de un análisis de senderos fue posible plantear un modelo con indicadores de ajuste óptimos, en el cual valores de tipo centralhumanista impactan directamente en el clima positivo y los indicadores de bienestar, mientras que valores de tipo social-humanista impactan directamente en el bienestar social. La evidencia obtenida es congruente con investigaciones previas que plantean la importancia de valores vinculados con la autonomía y el relacionamiento social óptimo para el funcionamiento adecuado de las personas y sus auto-evaluaciones.
\end{abstract}

Palabras clave: bienestar, valores individuales, clima emocional, violencia política

\begin{abstract}
The period of violence in Peru between 1980 and 2000 generated significant psychosocial sequelae in the population, mainly in people living in the highlands and the central jungle. Ayacucho, a region in the central highlands, has been identified as the one hit hardest by this process. The present study analyzed the relations among well-being, values, and emotional climate in 120 people who lived in Ayacucho during those years, selected through a purposive sampling method. Humanitarian motivation and central and social orientation values displayed a direct correlation with emotional climate and subjective and social well-being. A path analysis was used to generate a model with optimal goodness of fit in which central-humanistic values directly affect climate and well-being indicators, while socialhumanistic values have a direct impact on social well-being. The evidence obtained is in line with previous studies that stress the importance of values linked to autonomy and optimal social relationships for the proper functioning of individuals and their self-evaluations.
\end{abstract}

Keywords: well-being, individual values, emotional climate, political violence

El conflicto armado interno (CAI) que vivió el Perú entre los años 1980 y 2000 es considerado como uno de los procesos más violentos en toda su historia republicana. Esta afirmación se desglosa tanto por la cantidad de muertos y desaparecidos, como por las profundas secuelas que dejó en la sociedad peruana (Comisión de la Verdad y Reconciliación [CVR], 2003). Se dice que tales efectos pueden constatarse hasta el día de hoy tan solo viendo los índices de inseguridad ciudadana (Instituto de Defensa Legal, 2015; Instituto Nacional de Estadística e Informática, 2016), las profundas desigualdades horizontales entre distintos grupos (Puyana, 2015; Thorp, 2010), los niveles de desaprobación de las principales instituciones del estado (Instituto de Estudios Peruanos, 2016) y la poca disposición del gobierno para ejecutar las recomendaciones en torno a la reconciliación planteadas por la CVR (Defensoría del Pueblo, 2013).

Investigaciones en contextos de violencia colectiva reportan que estos procesos afectan directamente los niveles de bienestar de las personas y generan la aparición y el fortalecimiento de climas emocionales

Henry Raúl Guillén Zambrano y Rosa María Cueto Saldívar, Grupo de Psicología Política y Social, Departamento de Psicología, Pontificia Universidad Católica del Perú, Lima, Perú.

Este artículo forma parte de la tesis "Bienestar, valores y clima emocional en personas expuestas a violencia política y procesos de justicia transicional en Ayacucho" del Programa de Psicología, mención en Psicología Social, de la Pontificia Universidad Católica del Perú.

La correspondencia relativa a este artículo debe ser dirigida a Henry Guillén, Departamento de Psicología, Pontificia Universidad Católica del Perú, Av. Universitaria 1801, San Miguel, Lima 32, Perú. E-mail: hguillen@pucp.pe 
negativos en los lugares más afectados (Beristain, Páez, Rimé \& Kanyangara, 2010; Páez, Fernández \& Beristain, 2001; Shmotkin \& Lomranz, 1998). También se identifican cambios socioculturales, como afectación de las creencias sobre el mundo y los otros (Páez et al., 2001), radicalización de las diferencias intergrupales (Martínez Martínez, Paterna Bleda \& Gouveia, 2006) y disminución de valores universalistas y benevolentes (Basabe, Valencia \& Bobowik, 2011).

Los valores se establecen como estructuras direccionadas hacia el logro de distintas metas y su consecución se conectaría con mayores niveles de bienestar en las personas (Sagiv \& Schwartz, 2000). Asimismo, se han identificado relaciones directas entre el bienestar y el clima emocional en diversos contextos (Techio et al., 2011; Zubieta, Muratori \& Mele, 2012). Este grupo de hallazgos abre la pregunta sobre las posibles relaciones entre las variables mencionadas, más aún si previamente se enfatizó que la violencia colectiva las afecta directamente.

Con el fin de comprobar lo planteado, se llevó a cabo una investigación empírica en un grupo de personas que vivieron el contexto de violencia política en el Perú, específicamente en Ayacucho.

El artículo aborda de manera descriptiva los principales hallazgos respecto de los efectos psicosociales de la violencia política en el Perú y en otros casos. Luego, se presentan las variables de bienestar, valores y clima emocional, así como las principales relaciones que la evidencia empírica reporta hasta el momento. Posteriormente, se describen los principales resultados y, finalmente, se discute lo hallado, planteando algunas conclusiones y recomendaciones.

\section{Impacto Psicosocial de la Violencia Política: el Caso Peruano}

La violencia política acontecida por casi dos décadas en el Perú significó un impacto en duración y pérdidas aún mayor que los conflictos que el Estado peruano tuvo con países vecinos de Latinoamérica. En términos de magnitud, el número de víctimas mortales reportado por la CVR asciende a 23.969, entre muertos y desaparecidos, y se logró estimar en 69.280 las víctimas fatales, tomando en cuenta el método de sistemas múltiples (CVR, 2003).

El CAI evidenció las profundas brechas que perfilaban a la sociedad peruana, ya que las más afectadas fueron personas ubicadas en zonas significativamente menos atendidas por el Estado, específicamente, las zonas rurales y en condiciones de pobreza y pobreza extrema de la sierra y selva peruanas. Las cifras de la CVR (2003) revelaron que el 85\% de víctimas fatales pertenecía en primer lugar a la región de Ayacucho, seguida por las regiones de Junín, Huánuco, Huancavelica, Apurímac y San Martín. El 55\% de las víctimas fatales trabajaba en actividades agropecuarias en contextos rurales y un $75 \%$ tenía como lengua materna el quechua u otra lengua nativa.

Respecto de los desencadenantes de la violencia, se establece como punto de partida para el CAI la decisión del Partido Comunista del Perú-Sendero Luminoso de iniciar la "guerra popular" contra el Estado peruano. Sin embargo, es importante tomar en cuenta la influencia de factores contextuales e históricos que facilitaron el desarrollo del conflicto, entre los cuales se puede destacar: (a) la exclusión social, como retrato de una dinámica que visibilizó la frágil presencia del Estado en algunas regiones y la aun más débil identificación como colectivo nacional por parte de algunos grupos regionales; (b) la dificultad a nivel social para consolidar un proyecto nacional en el que sus distintos grupos fueran atendidos e incluidos de manera equitativa; c) la profunda crisis económica y política acontecida en los años 80 e inicios de los 90 y (d) la incapacidad de los gobiernos de turno para enfrentar y detener el avance de la violencia (Comas-Díaz, Lykes \& Alarcón, 1998; CVR, 2003; Espinosa, Schmitz \& Cueto, 2015).

Las secuelas psicosociales del CAI reportadas por la CVR (2003) fueron la instauración de emocionalidad negativa en la población afectada, tanto a nivel individual como colectivo, las relaciones interpersonales quedaron dominadas por la desconfianza y el miedo, las autoridades quedaron deslegitimadas por su falta de acción para la protección del colectivo, la estructura comunitaria quedó debilitada, y con ello las organizaciones locales y las redes de protección, se instauraron dinámicas de discriminación y estigma hacia la población rural, factor que afectó significativamente las identidades locales, generando pérdidas a nivel de tradiciones, costumbres y ritos. Dada la pérdida de padres u otros miembros de la estructura nuclear, a nivel familiar se perdieron fuentes de apoyo material e inmaterial y, al mismo tiempo, la convivencia intra-familiar se vio afectada. Muchas de estas secuelas, principalmente las de tipo emocional, siguieron reportándose aún después de algunos años de finalizado el CAI. 
Algunas investigaciones reportan impactos significativos en las personas directamente afectadas por la violencia política en rendimiento académico (Theidon, 1999) y sintomatología vinculada a estrés agudo, trastorno de estrés postraumático (TEPT), depresión, ansiedad y alcoholismo (CVR, 2003; Darghouth, Pedersen, Bibeau \& Rousseau, 2006; Kendall, Matos \& Cabra, 2006; Snider et al., 2004; Theidon, 1999).

La violencia política, tal como se retrata, generó heridas visibles, principalmente físicas, como también invisibles, que se relacionan con su impacto a nivel emocional, social y cultural, las que son menos evidentes.

$\mathrm{Al}$ respecto, Wessells y Kostelny (2011) plantean que los impactos de la violencia política son contextuales y no se distribuyen de manera igualitaria, por lo que su variabilidad dependerá del grado de exposición, de vulnerabilidad y de conectividad social o redes de soporte con los que cuente una persona. Así, se han identificado factores que aumentan la vulnerabilidad ante procesos de violencia política, tales como la etapa de desarrollo y las capacidades, el género — de acuerdo al contexto- o el nivel socioeconómico.

Los impactos psicosociales de la violencia política se pueden clasificar tomando en cuenta el foco de análisis: individual, social y/o cultural. En el plano individual, se identifican impactos severos referidos principalmente al ámbito clínico y psiquiátrico, como dificultades de sueño, desarrollo de sintomatología vinculada al TEPT, abuso de alcohol y drogas, como reacción no adaptativa al duelo, y depresión en distintos niveles (Levitt, 2011; Organización Mundial de la Salud [OMS], 2002; Wessells \& Kostelny, 2011).

A nivel social, se han identificado cambios significativos en el tejido social y las redes interpersonales, memorias colectivas cargadas de afectividad negativa, exacerbación de la pobreza de los grupos más afectados y dificultades para acceder a educación y empleo, desesperanza frente al futuro, polarización y conflicto intergrupal asentado en la radicalización de la lógica nosotros-ellos, deshumanización del exogrupo, desplazamiento interno o externo y violencia intrafamiliar e interpersonal (Bilali, 2011; Holtz \& Wagner, 2011; Wessells \& Kostelny, 2011).

A nivel cultural, las creencias y prácticas de cada contexto determinarán también los tipos y niveles de afectación que experimenta cada uno de sus miembros. Este punto permite tomar con pinzas la tipología occidental sobre las secuelas de la violencia, ya que el malestar psicológico experimentado por algunos contextos podría estar determinado por elementos vinculados, entre otros, a su espiritualidad, a un mayor foco en la armonía del colectivo, en comparación a un foco puramente individual, a la manera en que se experimenta el estrés y a la ritualización de las experiencias vinculadas a la muerte y al duelo (Wessells \& Kostelny, 2011).

Aun cuando los impactos difieren en tipo y grado, la evidencia ha ampliado el marco de afectación, saltando de lo clínico-psiquiátrico a indicadores que exploran el bienestar, así como variables colectivas y culturales a considerar. En el siguiente acápite se desarrollan las variables de bienestar, valores y clima emocional que fueron exploradas en el presente estudio.

\section{Bienestar, Valores y Clima Emocional}

El estudio del bienestar se sustenta en la psicología positiva y ahonda en los componentes positivos y potenciadores del ser humano y de los procesos que subyacen a la felicidad como fenómeno psicológico (Cuadra L. \& Florenzano U., 2003; Diener \& Ryan, 2009).

Así, el bienestar subjetivo (SWB), planteado a partir de la tradición hedónica del bienestar (Ryan \& Deci, 2001), se estructura en base a componentes cognitivos y afectivos vinculados con la satisfacción con la vida y el balance emocional, respectivamente (Diener \& Ryan, 2009). Ambos componentes han sido relacionados con un mayor y diverso número de experiencias sociales, alta motivación de logro e indicadores de buena conducta social (Ryan \& Deci, 2001; Sheldon et al., 2004).

Como variable social respecto del funcionamiento óptimo del individuo, se plantea el concepto de bienestar social (BS: Blanco \& Díaz, 2005; Keyes, 1998), con el que se analizan las relaciones individuo-medio, desde la valoración que el sujeto hace sobre su entorno, las dinámicas configuradas en la interacción y su funcionamiento en estos espacios. Estructuralmente, el BS se compone de cinco dimensiones: integración, aceptación, contribución, actualización y coherencia social.

Entre los factores determinantes del bienestar, algunas investigaciones vienen explorando las relaciones entre los valores y distintas medidas del bienestar (Becchetti, Bruni \& Zamagni, 2015; Bilbao, Techio \& Páez, 2007; Bobowik, Basabe, Páez, Jiménez \& Bilbao, 2011; Jamaludin, Sam, Sandal \& Adam, 2016; Sagiv \& Schwartz, 2000; Sortheix \& Lönnqvist, 2015). En términos generales, los valores se conceptualizan como 
creencias conectadas a emociones de carácter transituacional que se refieren a fines y conductas deseables, guían juicios respecto de las personas, comportamientos y sucesos y se ordenan jerárquicamente según la importancia relativa asignada por la persona, tomando en cuenta la cultura a la cual se está circunscrita, generando un sistema vertical capaz de establecer normas evaluativas, justificar conductas, expresar cognitivamente necesidades y ajustarse al medio (Bilbao et al, 2007; Gouveia, Milfont \& Guerra, 2014a; Schwartz et al., 2012).

Las relaciones entre el bienestar y los valores presentan resultados diversos que pueden organizarse en dos perspectivas: la de valores saludables y la de congruencia (Bobowik et al., 2011; Sagiv \& Schwartz, 2000). La primera perspectiva se basa en la teoría de la auto-determinación (Ryan \& Deci, 2001) y plantea que, en base al tipo de motivación antepuesto a los valores -intrínseca o extrínseca-, estos promoverían mayores o menores niveles de bienestar, siendo así que valores que reflejen crecimiento psicológico y auto-actualización cimentarían el bienestar, mientras que aquellos orientados hacia la aprobación y búsqueda de admiración de otros la dinamitarían. La segunda perspectiva plantea que el mantenimiento de valores congruentes con los del ambiente llevaría a mayores niveles de bienestar, por ende, la experiencia de bienestar estaría ajustada a la viabilidad contextual para la expresión de los valores individuales y el logro de metas. De esta forma, habría mayores oportunidades de satisfacción de necesidades individuales y bienestar en tanto los valores socialmente deseables se enlacen a las necesidades individuales, respondan o no a motivaciones intrínsecas o extrínsecas.

Diversas investigaciones han corroborado las hipótesis de valores saludables (Bilbao et al., 2007; Bobowik et al., 2011; Jamaludin et al., 2016; Sagiv \& Schwartz, 2000) y de congruencia (Oishi, Diener, Lucas \& Suh, 1999; Sagiv \& Schwartz, 2000; Sortheix \& Lönnqvist, 2015). Si bien muchos de los estudios mencionados se desarrollaron con poblaciones diversas que variaron según el lugar de nacimiento, la condición de vida en el país habitado y el nivel educativo, entre otras variables, no se ha logrado un consenso general sobre alguna de las perspectivas desarrolladas.

Además de lo anterior, investigaciones en contextos post violencia política refieren al clima emocional como una variable necesaria a explorar (Cárdenas, Páez, Rimé, Bilbao \& Asún, 2014; de Rivera, Kurrien \& Olsen, 2007; de Rivera \& Páez, 2007; Techio et al. 2011). El clima emocional se define como el conjunto de emociones percibidas como predominantes colectivamente, que son independientes de la emocionalidad personal y que emergen de la interacción social en determinados contextos y momentos históricos (de Rivera et al., 2007; de Rivera \& Páez, 2007). La predominancia de ciertas emociones colectivas funcionaría como un factor de influencia en la manera en que se desarrollan las relaciones interpersonales en el endogrupo (Techio et al., 2011). La clasificación de los climas emocionales se da en base a su percepción como positivos (seguridad, confianza, solidaridad, entre otros) o negativos (miedo, enojo, entre otros). Climas emocionales positivos se han relacionado con indicadores de desarrollo social (Techio et al., 2011).

Sobre las relaciones entre los valores y el clima emocional, de Rivera y Yurtsever (2010) hallaron nexos entre contextos con predominancia de valores igualitarios, baja distancia al poder, percepción de distribución igualitaria en las relaciones de poder, y climas emocionales positivos. Los hallazgos refuerzan la importancia de valores como la autonomía, la igualdad y el apoyo social para generar contextos socialmente menos estresantes y con mayores niveles de bienestar. Junto a esto, Techio et al. (2011) refieren relaciones directas entre BS y clima positivo y relaciones inversas entre clima negativo y SWB, lo que pone de relieve el valor de contextos en donde prevalecen condiciones de aceptación e integración interpersonal, además de la comprensión y control del medio como variables que facilitan la afectividad positiva en el colectivo (Bilbao et al., 2011; León Urteaga, 2012).

La evidencia en países que pasaron por periodos de violencia política refiere menores niveles de SWB en periodos prolongados, incluso en condiciones de seguridad, tanto para víctimas directas como indirectas de la violencia colectiva (Bilbao et al., 2011; Páez et al., 2001; Shmotkin \& Lomranz, 1998). Sobre el BS, estudios en Latinoamérica apuntan a que la percepción de violencia en el entorno se refiere a menores puntuaciones en BS, principalmente en integración, dimensión relacionada con la satisfacción con la vida y la afectividad positiva (Bilbao et al., 2011). Intragrupalmente, la exposición de los jóvenes a la violencia colectiva se ha relacionado con cambios interpersonales vinculados con la solidaridad y benevolencia endogrupal y un mayor compromiso con la ideología política dominante (Calderón Prada, Espinosa \& Techio, 2004; Conejero \& Etxebarria, 2007). Halloran (2007), además, encontró que valores igualitarios, universalistas y de baja conformidad funcionarían como predictores de la reconciliación intergrupal, especialmente ante identidades salientes en espacios de contacto intergrupal. Por último, investigaciones en contextos que experimentaron 
violencia colectiva reportan en las personas preponderancia de valores de orientación social, así como también valores de auto-trascendencia e indicadores de crecimiento psicológico (Cárdenas et al., 2014; Vázquez \& Páez, 2010).

\section{Objetivo e Hipótesis del Estudio}

Tomando en cuenta lo expuesto, el presente estudio tuvo como objetivo analizar las relaciones entre el SWB, el BS, los valores individuales y el clima emocional en un grupo de personas residentes en la región de Ayacucho, la región más afectada por la violencia política acontecida en el Perú durante los años 1980 y 2000.

En base a la evidencia empírica revisada preliminarmente y al contexto en el que se llevó a cabo el estudio, se planteó como hipótesis que los valores de motivación humanitaria y de orientación central y social tienen una influencia directa en el SWB, el BS y el clima emocional positivo (Bobowik et al., 2011; Halloran, 2007; Sagiv \& Schwartz, 2000).

\section{Método}

\section{Participantes}

La investigación contó con 120 participantes, todos adultos que residían en la provincia de Huamanga, de la región Ayacucho, en la sierra del Perú. Los principales criterios para seleccionar a los participantes fueron los siguientes: (a) haber nacido en Ayacucho, residir actualmente en la provincia de Huamanga y nunca haberse mudado de la región por un periodo mayor a seis meses y (b) ser mayores de 30 años, dado el periodo en el que ocurrió el CAI (1980-2000). Dichas características permitían asegurar que los participantes hubieran vivido en el contexto de violencia política.

Tomando en cuenta la escasez de estudios psicosociales realizados en la región señalada y que se encuentren vinculados con el tema de investigación, se determinó optar por una muestra no probabilística (Hernández Sampieri, Fernández Collado \& Baptista Lucio, 2010) de tipo intencional, con el fin de que a raíz de los hallazgos se puedan delimitar con mayor exactitud futuras investigaciones de mayor amplitud e intervenciones con población focalizada. Adicionalmente, se tuvieron en cuenta criterios para determinar el tamaño de la muestra para estudios no probabilísticos, principalmente respecto del número de sujetos por ítem de la escala (Argibay, 2009).

Conforme a lo planteado, las edades de las personas que participaron del estudio fluctuaron entre los 33 y 85 años $(M=45, D E=10,79)$. El $53,3 \%$ eran hombres y el $46,7 \%$, mujeres. El 40,8\% tenía como lengua materna el castellano y el $59,2 \%$, el quechua. Respecto del estado civil, el $55 \%$ de las personas participantes era casado/a, el $25 \%$ era soltero/a, el $13,3 \%$ era conviviente, el $2,5 \%$ era viudo/a, el $2,5 \%$ era separado/a y el 1,7\% era divorciado/a. En relación al nivel educativo, el 56,7\% tenía educación superior completa, el 10,8\%, educación superior incompleta, el 11,7\%, educación secundaria completa, el 7,5\%, educación técnica completa, el 1,7\%, educación secundaria incompleta, el 5,8\%, educación técnica incompleta, el 1,7\% educación primaria completa, el 3,3\%, educación primaria incompleta y el 0,8\%, educación inicial (pre-escolar). Finalmente, el $100 \%$ de los participantes vivió toda su vida en la provincia de Huamanga, en la región Ayacucho.

Sobre la exposición de los participantes a la violencia política, el $65 \%$ de ellos se percibía como víctima directa del periodo de violencia política y el $79,2 \%$ percibía que lo fueron sus familiares y amigos. Asimismo, consultados sobre el grado de exposición a la violencia, percibida en una escala Likert con extremos de 1 como nada expuesto y 6 como totalmente expuesto, el $82,5 \%$ de los participantes tuvo puntuaciones que fluctuaron entre 4 y $6(M=4,70, D E=1,30)$ para la exposición personal y el $82,6 \%$ tuvo puntuaciones que fluctuaron entre 4 y $6(M=4,75, D E=1,25)$ para el nivel de exposición de familiares y amigos.

\section{Instrumentos}

Datos demográficos. Se usó una ficha de datos demográficos que recopiló la edad de los participantes, el sexo, religión, lugar de residencia, actividades diarias y cantidad de años en la región.

Cuestionario de Valores Básicos (Gouveia, 2003). Se usó la versión en castellano de 18 ítems (CVB) de la propuesta de valores básicos de Valdiney V. Gouveia, derivada de su teoría funcionalista de los valores humanos (Gouveia et al., 2010, 2014a). Si bien existen propuestas como las de Schwartz (1994; Schwartz et 
al., 2012), Inglehart (1977) o Rokeach (1973), se optó por esta propuesta teórica debido a la capacidad para integrar distintas teorías, como las presentadas anteriormente, y generar una propuesta que articule los valores en base a dos funciones: su motivación (pragmático o idealista) y su orientación (social, central o personal). La integración de estas funciones genera una matriz de seis subfunciones: interactiva, suprapersonal, experimentación, normativa, existencia y realización. La presente propuesta ha sido debatida en su momento (Schwartz, 2014), recibiendo respuesta por el autor (Gouveia, Milfont \& Guerra, 2014b), quien ha resaltado el potencial parsimonioso e integrador de su teoría, así como la estructura de seis subfunciones que ha sido verificada en diversos contextos (Gouveia et al., 2010, 2014a; Gouveia, Vione, Milfont, Fischer, 2015).

El cuestionario plantea dos descripciones para cada valor (e.g., Tradición: a) Seguir las normas sociales de su país, b) Respetar las tradiciones de su sociedad). Para cada descripción de los valores, las puntuaciones se dan en una escala Likert de 7 puntos, siendo $1=$ Totalmente sin importancia y $7=$ De máxima importancia. La consistencia interna de la subfunción existencia, evaluada mediante alfa de Cronbach, fue 0,44, de la subfunción suprapersonal fue 0,62 , de la subfunción realización fue 0,40 , de la subfunción experimentación fue 0,42 , de la subfunción interactiva fue 0,47 y de la subfunción normativa fue 0,46 . Como se ha planteado, los indicadores obtenidos se alinean con aquellos obtenidos en estudios de los valores en general (Schwartz, 2005), en estudios previos usando la escala (Gouveia et al., 2010; Gouveia et al., 2014a) y en estudios realizados en el Perú (Seminario Obando, 2014).

Escala de Satisfacción con la Vida (Diener, Emmons, Larsen \& Griffin, 1985). Se usó la versión en castellano de la escala de Satisfacción con la Vida (SWLS) adaptada y empleada en diversos estudios en el Perú (Arellano Martorellet, 2011; Cueto, Espinosa, Guillén \& Seminario, 2016; Espinosa \& Tapia, 2011). El instrumento tiene cinco ítems que evalúan la satisfacción de la persona respecto de su vida (e. g., Estoy satisfecho con mi vida). La escala de respuesta es de 7 puntos, donde $1=$ No, en absoluto hasta $7=S i$, totalmente. Se obtuvo como consistencia interna un alfa de Cronbach de 0,71.

Escala de Balance Emocional. Se usó la versión adaptada al español de la escala de Afecto Positivo y Negativo (PANAS) de Watson, Clark y Tellegen (1988), usada en investigaciones en el Perú (Gargurevich \& Matos, 2012). Fue validada en estudiantes universitarios. La escala se compone de 20 ítems que miden la afectividad positiva (e.g., Entusiasmado/a) y negativa (e.g., Disgustado/a) que la persona experimentó en el último mes. El formato de respuesta Likert de cinco puntos va desde $1=$ Nada o casi nada hasta $5=$ Muchísimo. Se obtuvieron alfas de Cronbach de 0,52 para el afecto positivo y 0,57 para el negativo.

Escala de Bienestar Social (Keyes, 1998). Se usó la adaptación hecha por Arellano Martorellet (2011) de la versión adaptada y validada de Blanco y Díaz (2005). Dicha adaptación fue llevada a cabo para población peruana rural castellano-hablante: en una fase piloto del instrumento original se analizó la comprensión de cada ítem, reduciendo la extensión del instrumento, lo que facilitó el acceso y comprensión de dicha versión por parte de los participantes. El instrumento adaptado cuenta con 15 ítems (e.g., Me siento cercano a otra gente) que se puntúan en una escala Likert de 1 a 5 , siendo $1=$ Completamente en desacuerdo y $5=$ Completamente de acuerdo. Para el presente estudio se obtuvo un alfa de Cronbach general de 0,82 . Se tomaron en cuenta todas las dimensiones, considerando los criterios planteados por Mezulis, Abramson, Hyde y Hankin (2004) y los resultados obtenidos en estudios en el Perú (Arellano Martorellet, 2011; Balbuena Blengeri, 2013; Cueto et al., 2016) con la misma escala.

Escala de Clima Emocional (Páez et al., 1997). Se usó la versión de 10 ítems que se organiza en ocho ítems que se refieren a la percepción de emociones básicas a nivel colectivo (e.g., El ambiente o clima social es: De esperanza, esperanzado) y dos que se refieren a la percepción de la situación económica y el clima afectivo en general. Dicha versión se llevó a cabo en muestras de seis países, incluido el Perú. El instrumento cuenta con validez convergente. Todos los ítems se refieren al estado actual del país. La escala consta de dos factores: clima emocional positivo y clima emocional negativo. Las opciones de respuesta se componen de 5 puntos y van desde $1=N a d a$ a $5=$ Mucho. Para el presente estudio se obtuvo un alfa de Cronbach de 0,76 para el clima positivo y 0,71 para el clima negativo. La escala probada en población peruana obtuvo una consistencia interna similar (Ferrándiz, 2011; León, 2012; Ordinola Macha, 2012).

Experiencia de victimización respecto al CAI. Se hicieron las siguientes preguntas: (a) ¿Se considera usted una víctima de la violencia durante los años 1980-2000?, (b) ¿Algunos de sus familiares o amigos cercanos han sido víctimas de la violencia durante los años 1980-2000?, (c) En una escala del 1 al 6, donde 
1 es Casi Nada y 6 Totalmente, ¿Cuál cree usted que fue su grado de exposición a la violencia durante el periodo 1980-2000? y (d) En una escala del 1 al 6, donde 1 es Casi Nada y 6 Totalmente, ¿Cuál cree usted que fue el grado de exposición de sus familiares y amigos cercanos a la violencia durante el periodo 1980-2000?

\section{Procedimiento}

Se realizaron visitas a la región de Ayacucho, específicamente a la provincia de Huamanga, donde se aplicaron los instrumentos. La aplicación se hizo de manera individual y se accedió a los participantes de acuerdo a su disponibilidad. La aplicación fue de 40 minutos, en promedio, por participante. De acuerdo a la preferencia del participante, los cuestionarios se aplicaron en sus hogares, en la mayoría de los casos, o en lugares públicos establecidos con ellos/as. La información fue recogida en un tiempo aproximado de dos meses.

Previamente al llenado del cuestionario, se informó a los/as participantes sobre la investigación y sus objetivos mediante un consentimiento informado, el cual leyeron y firmaron antes de empezar con el llenado de los protocolos.

\section{Análisis de Datos}

Se analizaron los datos usando estadísticos descriptivos y evaluando las correspondientes pruebas de normalidad y pruebas de consistencia con alfa de Cronbach. También se llevaron a cabo análisis correlacionales y de diferencias de medias, tanto en sus versiones paramétricas y no paramétricas, según correspondiera a la distribución de datos de cada variable evaluada. Con el fin de comparar las diferencias obtenidas entre los factores del balance emocional, se usó la prueba de Wilcoxon para dos muestras dependientes. Para comparar las diferencias obtenidas en las subfunciones de los valores se usó la prueba de Friedman para más de dos muestras dependientes. Para este propósito se usó SPSS. Cabe resaltar que no se realizaron análisis factoriales para ninguna de las escalas; se tomaron en cuenta las dimensiones teóricas ratificadas en estudios nacionales.

Usando el programa AMOS, se llevaron a cabo análisis de senderos con el fin de evaluar las propiedades de modelos hipotéticos propuestos. Como criterios para evaluar el ajuste del modelo a los datos se usaron los siguientes indicadores: $\chi^{2} / g l$, el índice comparativo de Bentler-Bonett (CFI), el índice normalizado de BentlerBonett (NFI) y la raíz del error cuadrático medio de aproximación (RMSEA) de Steiger-Lind. Se consideraron como valores mínimos de un adecuado ajuste: $\chi^{2} / g l<3$, CFI y NFI $>0,90$ y RMSEA $<0,08$ (Batista-Foguet \& Coenders, 2000; Kline, 2005; Ruiz, Pardo \& San Martín, 2010).

\section{Resultados}

\section{Descriptivos de las Variables}

De manera descriptiva, los resultados en la Tabla 1 refieren puntuaciones medias y altas en las distintas subfunciones de los valores. Sin embargo, la subfunción existencia resultó mayor que el resto de las subfunciones, $Q=233,60, p<0,001$. En relación al BS, se reportan puntajes altos para el puntaje global. Respecto del SWB, se reportan puntajes altos en la satisfacción con la vida. Sobre el balance emocional, los resultados mostraron que el balance emocional positivo sería mayor que el negativo, $Z=-7,09, p<0,001$. En torno al clima emocional, no se obtuvieron diferencias entre el clima positivo y negativo, $Z=-0,402, p=0,688$.

\section{Relaciones entre las Medidas de Bienestar, Valores y Clima Emocional}

Como se muestra en la Tabla 2, se presentan correlaciones de efectos medianos, según Cohen (1988), entre algunas subfunciones de los valores y el clima emocional positivo con distintas medidas del SWB y BS. Así el puntaje total del BS, la dimensión de integración y la de contribución social se correlacionaron con valores interactivos y suprapersonales, así como con el clima emocional positivo. Además, la satisfacción con la vida se correlacionó con el clima positivo y los valores suprapersonales. Por otro lado, el clima positivo se correlacionó con el balance emocional positivo. En contraposición, el clima negativo se correlacionó inversamente con la satisfacción con la vida. 
Tabla 1

Descriptivos de las Variables

\begin{tabular}{|c|c|c|c|c|c|c|c|c|c|}
\hline \multirow{2}{*}{ Variable } & \multirow{2}{*}{ Instrumento } & \multirow{2}{*}{ Medida/Dimensión } & \multicolumn{3}{|c|}{ Descriptivos } & \multicolumn{4}{|c|}{ Normalidad } \\
\hline & & & $M$ & $M e$ & $D E$ & $Z$ & $p$ & g1 & g2 \\
\hline \multirow[t]{6}{*}{ Valores } & CVB & Existencia & 5,68 & 5,67 & 0.67 & 0,14 & 0,001 & $-0,96$ & 4,44 \\
\hline & CVB & Suprapersonal & 5,26 & 5,33 & 0,78 & 0,11 & 0,001 & $-0,19$ & $-0,01$ \\
\hline & CVB & Realización & 4,53 & 4,50 & 0,96 & 0,14 & 0,001 & $-0,37$ & 0,77 \\
\hline & CVB & Experimentación & 4,25 & 4,33 & 0,78 & 0,14 & 0,001 & $-0,37$ & 0,99 \\
\hline & CVB & Interactiva & 5,40 & 5,50 & 0,91 & 0,18 & 0,001 & $-1,22$ & 4,10 \\
\hline & CVB & Normativa & 5,26 & 5,33 & 0,84 & 0,12 & 0,001 & $-0,44$ & 0,21 \\
\hline \multirow[t]{4}{*}{ Bienestar } & Keyes & Bienestar social & 3,69 & 3,73 & 0,62 & 0,09 & 0,024 & $-0,54$ & $-0,13$ \\
\hline & SWLS & Satisfacción con la vida & 4,70 & 4,80 & 1,03 & 0,09 & 0,024 & $-0,30$ & $-0,38$ \\
\hline & PANAS+ & Balance emocional positivo & 2,85 & 2,80 & 0,45 & 0,09 & 0,024 & 0,23 & 0,60 \\
\hline & PANAS- & Balance emocional negativo & 2,54 & 2,50 & 0,46 & 0,13 & 0,001 & 0,79 & 0,43 \\
\hline \multirow[t]{2}{*}{ Clima emocional } & Páez et al. & Clima emocional positivo & 2,84 & 2,83 & 0,63 & 0,08 & 0,098 & 0,20 & $-0,30$ \\
\hline & Páez et al. & Clima emocional negativo & 2,88 & 3,00 & 0,77 & 0,09 & 0,008 & 0,17 & $-0,04$ \\
\hline
\end{tabular}

g1 = Asimetría, g2 = Curtosis

Con el fin de analizar las variables de manera integrada, se llevó a cabo un análisis de senderos tomando como base la literatura que relaciona los valores individuales, el SWB, el BS y el clima emocional (Becchetti et al., 2015; Bilbao et al., 2007; Bilbao et al., 2011; Bobowik et al., 2011; de Rivera \& Yurtsever, 2010; Jamaludin et al., 2016; Sagiv \& Schwartz, 2000; Sortheix \& Lönnqvist, 2015; Techio et al., 2011). Importante es recalcar que, si bien la literatura todavía no ha llegado a consenso respecto del tipo de valores que estarían a la base de mayores niveles de bienestar, ya que estos pueden ser intrínsecos o contextuales, se propuso un modelo hipotético (ver Figura 1), tomando en cuenta tanto los resultados obtenidos a nivel correlacional, la evidencia en contextos post violencia política y la recurrencia de cierto tipo de valores que han sobresalido en investigaciones para validar las distintas propuestas.

Como se muestra en la Tabla 3, el modelo hipotético propuesto logró indicadores de ajuste adecuados. No obstante, se obtuvieron estimadores no significativos en muchas de las relaciones entre los valores respecto al bienestar y el clima emocional, específicamente en aquellas que involucraron a valores de las subfunciones de experimentación e interactiva. En vista de ello, se propone un modelo alternativo (ver Figura 2) que cumple con los criterios de ajuste y relacionamiento óptimo entre las variables.

Como se evidencia en la Tabla 3 , el modelo alternativo obtuvo similares indicadores de ajuste respecto del modelo anterior. Sin embargo, los niveles de relación entre las variables mejoraron, resultando todas las correlaciones significativas. Por tal motivo, no hubo necesidad de realizar nuevos ajustes. El modelo planteado refiere que: (a) los valores de orientación central y de motivación humanitaria, expresados en la subfunción suprapersonal, incidirían directamente en la satisfacción con la vida, el balance emocional positivo, el BS y el clima emocional positivo y (b) los valores de orientación social y de motivación humanitaria, expresados en la subfunción interactiva, incidirían directamente en el BS. Las relaciones identificadas se alinean con las obtenidas en investigaciones previas, tanto para las relaciones entre valores y bienestar (Bilbao et al., 2007; Bobowik et al., 2011; Jamaludin et al., 2016; Sagiv \& Schwartz, 2000) como para las relaciones entre valores y clima emocional (de Rivera \& Yurtsever, 2010).

Así, también, se establecen relaciones entre las diferentes medidas de bienestar, similares a la evidencia previa en contextos nacionales (Arellano Martorellet, 2011; Balbuena Blengeri, 2013; Cueto et al., 2016), entre la afectividad colectiva y los diferentes tipos de bienestar y también entre las subfunciones de valores propuestas en el modelo (Gouveia et al., 2010; Gouveia et al., 2014a). 
Tabla 2

Correlaciones entre las Variables de Estudio

\begin{tabular}{|c|c|c|c|c|c|c|c|c|c|c|c|c|}
\hline & 1 & 2 & 3 & 4 & 5 & 6 & 7 & 8 & 9 & 10 & 11 & 12 \\
\hline 1. Valores existencia & 1 & $0,30 * *$ & $0,45^{* *}$ & $0,37 * *$ & $0,43^{* *}$ & $0,43 * *$ & 0,14 & $0,18^{*}$ & 0,17 & 0,04 & 0,13 & 0,05 \\
\hline 2. Valores normativa & & 1 & $0,36^{* *}$ & $0,22^{*}$ & $0,30 * *$ & $0,31^{* *}$ & 0,12 & 0,10 & 0,08 & 0,01 & 0,07 & 0,10 \\
\hline 3. Valores suprapersonal & & & 1 & $0,27^{* *}$ & $0,47^{* *}$ & $0,39 * *$ & $0,31^{* *}$ & $0,33^{* *}$ & $0,21^{*}$ & 0,12 & $0,24^{* *}$ & $-0,07$ \\
\hline 4. Valores experimentación & & & & 1 & $0,40 * *$ & $0,25^{* *}$ & $0,23^{*}$ & $0,21^{*}$ & 0,02 & $-0,04$ & $0,26^{* *}$ & $-0,11$ \\
\hline 5. Valores realización & & & & & 1 & $0,27 * *$ & 0,11 & 0,15 & 0,15 & 0,05 & 0,16 & $-0,07$ \\
\hline 6. Valores interactiva & & & & & & 1 & $0,32^{* *}$ & $0,21^{*}$ & 0,15 & 0,13 & 0,14 & 0,08 \\
\hline 7. Bienestar social & & & & & & & 1 & $0,46^{* *}$ & 0,05 & $-0,11$ & $0,37 * *$ & $-0,18$ \\
\hline 8. Satisfacción con la vida & & & & & & & & 1 & 0,13 & $-0,10$ & $0,44^{* *}$ & $-0,27 * *$ \\
\hline 9. PANAS positivo & & & & & & & & & 1 & $0,63^{* *}$ & $0,30^{* *}$ & 0,04 \\
\hline 10. PANAS negativo & & & & & & & & & & 1 & 0,08 & 0,14 \\
\hline 11. Clima positivo & & & & & & & & & & & 1 & $-0,34^{* *}$ \\
\hline 12. Clima negativo & & & & & & & & & & & & 1 \\
\hline
\end{tabular}




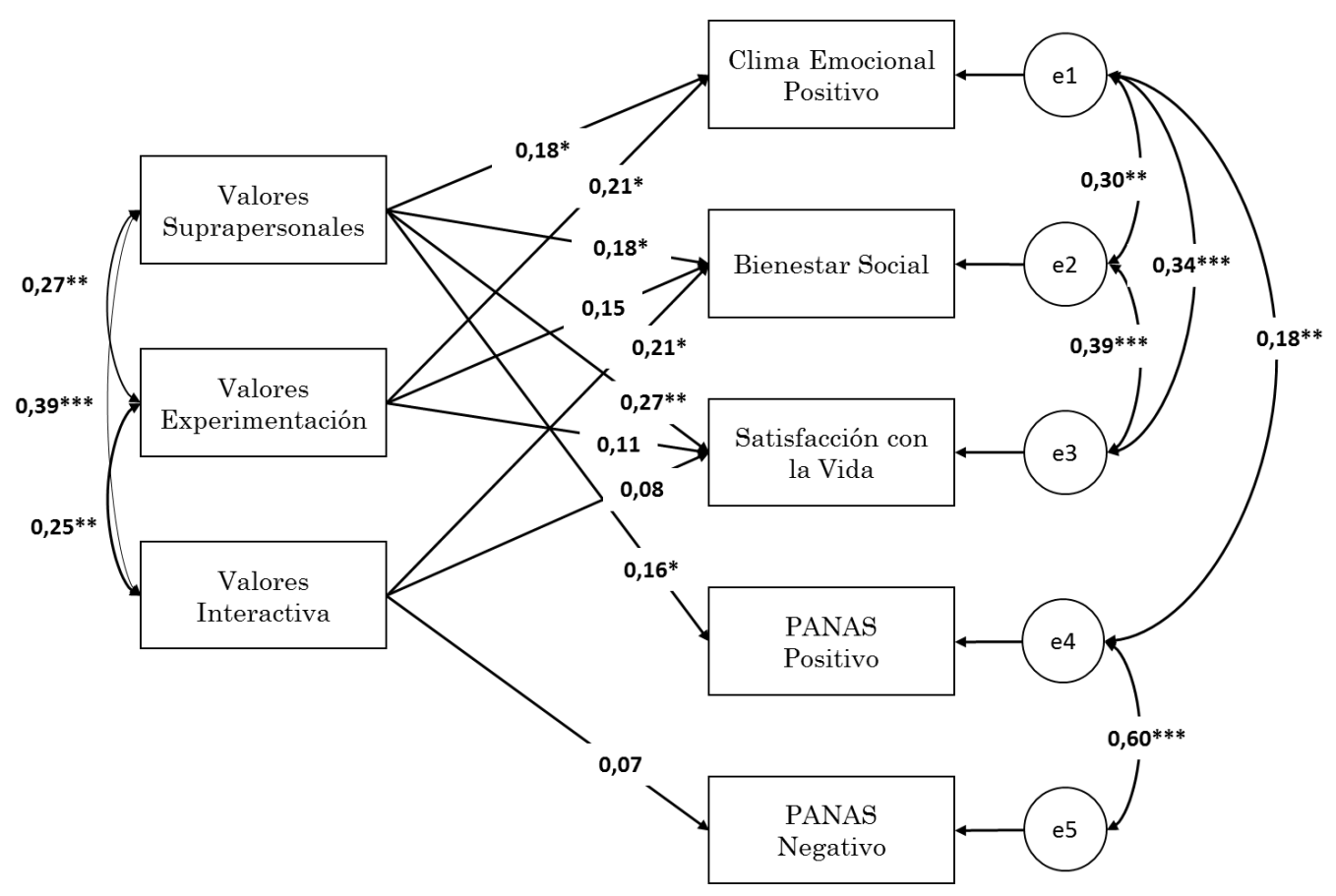

Figura 1. Análisis de senderos del modelo hipotético. ${ }^{* * *} p<0,001,{ }^{* *} p<0,01,{ }^{*} p<0,05$.

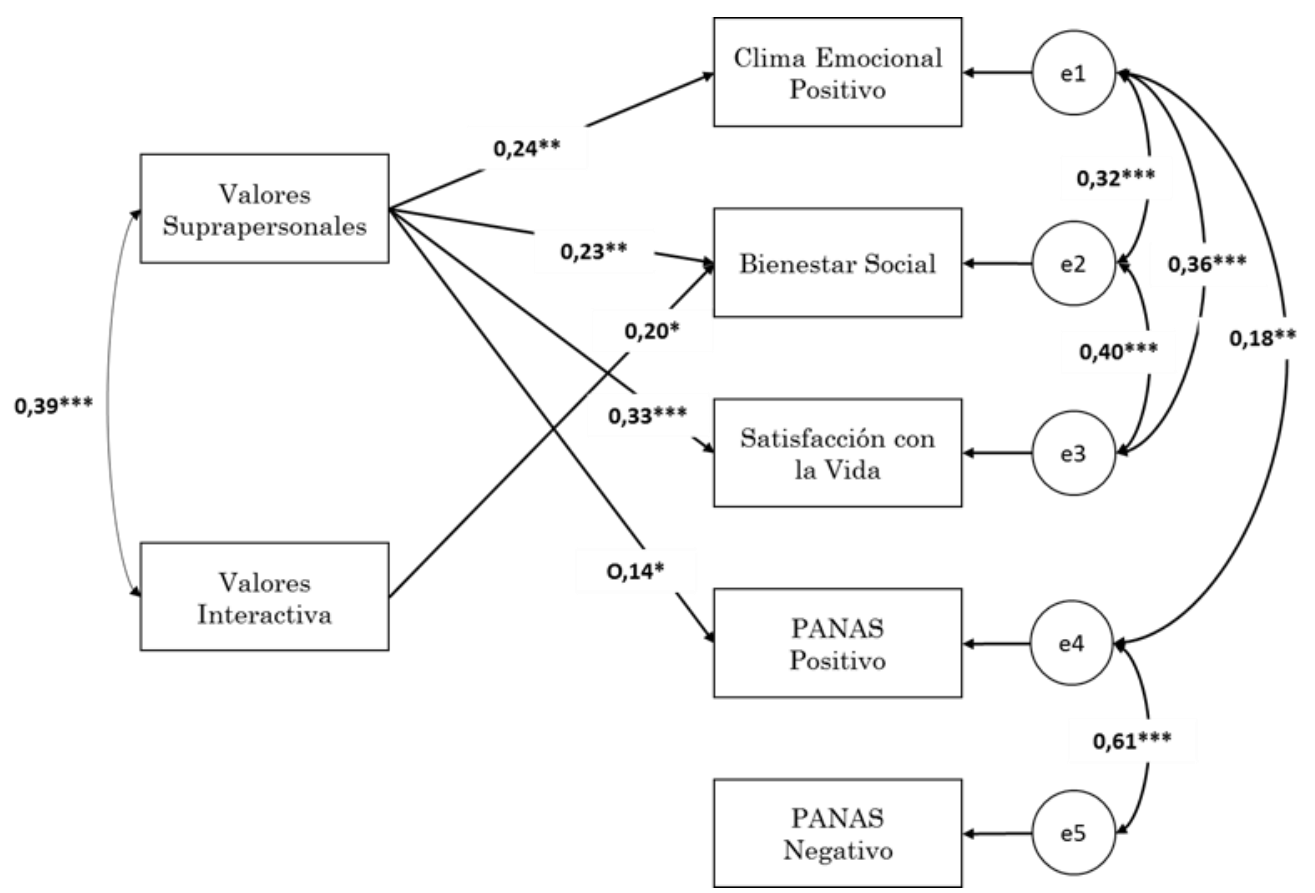

Figura 2. Análisis de senderos del modelo alternativo. ${ }^{* * *} p<0,001 ; * * p<0,01$; ${ }^{*} p<0,05$. 
Tabla 3

Índices de Bondad de Ajuste del Análisis de Senderos

\begin{tabular}{lcccc}
\hline & $\chi^{2 / g l}$ & CFI & NFI & RMSEA \\
\hline Modelo hipotético & 1,53 & 0,97 & 0,93 & 0,07 \\
Modelo alternativo & 1,63 & 0,96 & 0,91 & 0,07 \\
\hline
\end{tabular}

\section{Discusión y Conclusiones}

\section{Bienestar, Valores Individuales y Clima Emocional}

A nivel descriptivo, los resultados revelan niveles de bienestar altos en sus distintas mediciones. Respecto del BS, se obtuvieron puntuaciones altas a nivel global. Si bien no se tienen mediciones previas de los participantes del estudio que permitan realizar comparaciones y ser más concluyente, se podría tomar en cuenta lo planteado por la CVR (2003) sobre las secuelas psicosociales identificadas y plantear que a nivel de funcionamiento social podrían estarse generando o fortaleciendo procesos de reestructuración y mayor vinculación colectiva. Esto último podría ser así, si se toma en cuenta que a nivel de características generales se percibían niveles de victimización individual y grupal por encima de la media. Como plantean Bilbao et al. (2011) respecto del BS, su medición permite explorar la vivencia de aspectos de cambio principalmente positivos, la percepción de menores problemas sociales — lo que incluye la violencia social—, menor anomia social y un clima emocional principalmente positivo.

Dada la naturaleza multidimensional de esta variable, se identificó que las dimensiones de contribución y coherencia social tuvieron puntajes mayores respecto de las demás. Puntuaciones altas en contribución social están relacionadas a sentimientos de utilidad, valoración del aporte personal hacia el endogrupo, mayor autoeficacia y mayor confianza respecto a la capacidad de organizar y ejecutar acciones socialmente relevantes (Bilbao et al., 2011; Blanco \& Díaz, 2005; Keyes, 1998). Adicionalmente, puntuaciones altas en la dimensión de coherencia social implican una mayor capacidad para entender la dinámica y funcionamiento social y una mayor comprensión y control del medio (Bilbao et al., 2011; Blanco \& Díaz, 2005; Keyes, 1998). Estos resultados contribuyen al análisis de la negativa hacia el resurgimiento de la violencia reportado en distintos entornos con afectación similar y en los que se reportan disposiciones favorables hacia la paz (Beristain et al., 2010).

Sobre el SWB, la satisfacción con la vida y el balance emocional fueron positivos. Como se menciona en estudios citados, dichas puntuaciones serían congruentes con contextos donde se percibiría un mayor número de espacios de memoria, mecanismos de reclamo y empoderamiento ciudadano (Beristain et al., 2010; Bilbao et al., 2011; Larisgoitia et al., 2009). Aunque dichos espacios no satisfagan las expectativas de la población afectada, el que se hayan podido estructurar podría aportar a la sensación de reconocimiento de lo vivido. Como mencionan Bilbao et al. (2011), tras procesos de violencia colectiva, a largo plazo la satisfacción con la vida tiende a normalizarse, brindando puntuaciones medianamente altas, y no estaría relacionada con niveles de calidad de vida, incluso en el caso de víctimas directas de la violencia. Reforzando esta idea, Shmotkin y Lomranz (1998) no encontraron diferencias en SWB entre víctimas directas e indirectas del Holocausto nazi. Estos resultados se alinean con los reportes sobre crecimiento post-traumático reportado en estudios en contextos latinoamericanos (Cárdenas et al., 2014; Vázquez \& Páez, 2010), así como reportes en otros países donde se realizaron procesos de justicia transicional (Beristain et al., 2010; Cilliers, Dube \& Siddiqi, 2016).

Respecto de los valores, el estudio reveló puntuaciones altas para las subfunciones de orientación central, existencia y suprapersonal, y para valores de orientación social, interactiva. La subfunción existencia representa necesidades fisiológicas básicas y de seguridad, siendo su propósito principal garantizar las condiciones básicas de supervivencia biológica y psicológica del individuo (Gouveia et al., 2010; Gouveia et al., 2014a). La evidencia empírica en contextos post violencia política plantea que entre las principales necesidades a satisfacer se encontrarían la supervivencia y la seguridad, especialmente en contextos económicos y sociales precarios (Basabe et al., 2011; Bilbao et al., 2007; Bobowik et al., 2011; Schwartz et al., 2012). Cabe mencionar que, aunque pasados casi 20 años del proceso de violencia política, las condiciones económicas y sociales, principalmente en los contextos más afectados, aún son precarias, además de que el 
proceso de reconciliación no se evidencia como prioridad para los gobiernos que secundaron el informe de la CVR (Barrenechea, 2010; Defensoría del Pueblo, 2013). Los valores de la subfunción suprapersonal, que responde a necesidades estéticas, de cognición y de autorrealización, se centran en orientaciones de carácter universalista que facilitan la categorización del mundo de manera consistente, facilitando la organización cognitiva (Gouveia et al., 2010; Gouveia et al., 2014a). El hecho de que estos valores resalten en los participantes estaría relacionado con la necesidad que emerge en estos procesos post-violencia por reconstruir y organizar de manera clara el mundo, dado que el tejido social ha sido significativamente resquebrajado (Bilbao et al., 2007). Dadas las raíces social y humanitaria de la subfunción interactiva, los resultados permiten acentuar la idea de que en contextos post-violencia se hace preponderante la satisfacción de necesidades vinculadas con la filiación y la pertenencia, pues estaría a la base una búsqueda por reconstruir el tejido afectado significativamente por el CAI, lo que lleva a estructurar el contacto interpersonal a nivel endogrupal como una meta en sí misma (Bilbao et al., 2007; Cárdenas et al., 2014; CVR, 2003; Gouveia et al., 2014a; Schwartz et al., 2012; Tay \& Diener, 2011; Vázquez \& Páez, 2010).

Respecto del clima emocional, no se evidenció diferencia entre los dos tipos de factores. No obstante, como plantean estudios previos (de Rivera \& Páez, 2007; de Rivera \& Yurtsever, 2010), la violencia colectiva devendría en el fortalecimiento de emocionalidad negativa a nivel grupal. Estos indicadores podrían mantenerse en el tiempo, en caso de que la población más afectada perciba discriminación y olvido por parte de sus instituciones y las políticas públicas elaboradas sobre los procesos de justicia y reconciliación (Lykes, Beristain \& Cabrera Pérez-Armiñan, 2007; Techio et al., 2011). Es importante resaltar aquí lo institucional, dado que la confianza en las instituciones se configuró como el puntaje más alto respecto de los demás climas que conforman los factores de dicha variable. Por tanto, existen factores coyunturales que afectan los climas emocionales, por lo que la situación del proceso de reconciliación (Defensoría del Pueblo, 2013) funcionaría como un elemento que incidiría de manera negativa en la afectividad colectiva.

\section{Relaciones Entre el Bienestar, los Valores Individuales y el Clima Emocional}

Además de los resultados de cada variable de manera independiente, el presente estudio reveló relaciones entre algunos tipos de valores individuales y las medidas de bienestar y clima emocional. Dicho relacionamiento mantiene coherencia con evidencia reportada previamente en países como Israel, Alemania (Sagiv \& Schwartz, 2000), España, Brasil, México, Chile y Ecuador, entre otros (Bilbao et al., 2007; Bobowik et al., 2011).

Como plantea el modelo propuesto en el análisis de senderos, valores vinculados a motivos humanitarios y de orientación central, como son los de la subfunción suprapersonal, funcionaron como predictores significativos del SWB en sus dos dimensiones, el BS y el clima emocional. Por otro lado, valores relacionados a motivos humanitarios y de orientación social, como son los de la subfunción interactiva, funcionaron como predictores significativos del BS.

Sobre lo suprapersonal, la evidencia empírica revisada refiere que valores dirigidos a la búsqueda de autonomía, madurez y organización cognitiva se relacionarían al bienestar. Esto podría deberse a que este tipo de valores facilita una orientación positiva hacia la diversidad, favorecería relaciones interpersonales positivas, fortalecería la percepción de un entorno integrado, evaluándolo de manera positiva, y se consideraría la libertad para contribuir y generar una visión eficaz del medio (Bilbao et al., 2007; Bobowik et al., 2011; Sagiv \& Schwartz, 2000). Como mencionan Bilbao et al. (2007), valores relacionados con la búsqueda de autonomía se vinculan a criterios de salud mental, lo que podría deberse a que están direccionados por motivación intrínseca, reforzando, así, el control, la autoeficacia y la autoestima (Ryan \& Deci, 2001). En la misma línea, Sagiv y Schwartz (2000) plantean que este tipo de valores reflejarían crecimiento psicológico y auto-actualización. Valores de este tipo, junto con la preocupación por el endogrupo, la estimulación y el logro, son denominados como valores que representarían necesidades de crecimiento (Bilsky \& Schwartz, 1994). La relación entre los valores suprapersonales y las dimensiones cognitiva y afectiva del SWB resultan sustanciales, puesto que resultados usando otros modelos (Schwartz et al., 2012) no reflejan este tipo de asociación en sus dimensiones símiles, por lo que la orientación central del modelo teórico planteado (Gouveia et al., 2010; Gouveia et al., 2014a) cobraría importancia, en tanto articula dos orientaciones extremas y se establece —en el presente estudio - como el principal predictor de distintas medidas de bienestar y de afectividad colectiva.

Sobre lo interactivo, los estudios revisados plantean que valores vinculados a la preocupación por el endogrupo y por la necesidad de establecer relaciones interpersonales positivas, íntimas y afectivamente 
satisfactorias estarían asociados al BS. Esto podría corroborar el carácter significativamente social de las personas, en tanto se encuentran predispuestas a integrarse y establecer vínculos positivos con el medio y sus miembros, así como por la necesidad de generar relaciones recíprocas y de utilidad endogrupal (Bilbao et al., 2007; Gouveia et al., 2014a; Schwartz et al., 2012). Como plantean Bilbao et al. (2007), la relación entre el bienestar y este tipo de valores posiblemente se sustenta en la orientación de estas creencias hacia una visión positiva del mundo social y de las relaciones interpersonales.

Los valores de autonomía y los valores dirigidos al bienestar del endogrupo y de las personas en general han sido asociados con metas y necesidades intrínsecas en el marco de la teoría de la autodeterminación (Ryan \& Deci, 2001). Esto es así, debido a que las necesidades de autonomía, relacionamiento y competencia son necesidades psicológicas básicas, cuya búsqueda lleva directamente a una satisfacción psicológica intrínseca. Siendo así, personas dirigidas por valores que responden a necesidades extrínsecas al sujeto evidencian niveles de bienestar menores, explorados en mediciones de auto-actualización, ansiedad, vitalidad, depresión y daño físico (Sheldon \& Kasser, 1995).

Las asociaciones obtenidas entre el bienestar y el clima emocional encuentran cierta congruencia, dado que el clima positivo se planteó en términos relacionales respecto al SWB y BS. Sobre esto, Páez et al. (2001) plantean que la percepción de un clima positivo podría sentar la base de un posterior crecimiento postviolencia. Así, la percepción de alegría, solidaridad, tranquilidad y esperanza en el colectivo estarían asentados en la percepción de cohesión, empatía y sensibilidad respecto de lo acontecido. Como mencionan Zubieta et al. (2012), los climas negativos estarían asociados a bajos niveles en aceptación, actualización y coherencia social, mientras que climas positivos serían reforzados por dimensiones como integración, aceptación y actualización social. La satisfacción con la vida se vincularía específicamente con climas positivos, como la esperanza, debido a que dichas construcciones favorecerían la percepción del entorno como seguro y equitativo (Ordinola Macha, 2012).

\section{Conclusiones, Limitaciones y Proyecciones}

El presente estudio buscó explorar las relaciones entre el bienestar, los valores y el clima emocional en un contexto que fue azotado por la violencia política acontecida en el Perú entre los años 1980 y 2000. Sobre esta base, se identificaron, a nivel descriptivo, niveles altos en las variables del estudio vinculadas al bienestar. Si bien no se contó con mediciones previas para realizar los contrastes respectivos, si se toma en cuenta la información remitida por la CVR (2003), podría plantearse que algunos procesos de crecimiento post-traumático se estarían evidenciando tras más de 15 años de culminado el periodo de violencia. Explorando en detalle, el nivel de confianza en las instituciones sería una de las variables que incidiría directamente en este resultado, dados los reportes de la Defensoría del Pueblo (2013) respecto de la situación del proceso de reconciliación en el Perú, en los que queda evidente la falta de interés político por llevar a cabo las recomendaciones que dejó la CVR.

A nivel contextual, resultados como los obtenidos en el presente estudio permiten evidenciar la necesidad por parte de personas que vivieron contextos de violencia de reestructurar el tejido social y dar sentido a los hechos ocurridos durante los años del CAI.

Por último, el presente estudio presentó algunas limitaciones a considerar en investigaciones futuras. Primero, la muestra no contempló la participación de población del entorno rural. El motivo principal fue que no se contaba con instrumentos adaptados y que hubieran sido previamente evaluados en estos contextos, donde prima una gran cantidad de población quechua-hablante. Futuras investigaciones podrían explorar dichas variables en este tipo de contextos, ya que fueron los más afectados por el CAI. Segundo, la cantidad de participantes y el tipo de muestreo no permiten realizar conclusiones más enfáticas, principalmente respecto del modelo propuesto, por lo que se abren nuevas posibilidades para que futuras investigaciones vinculadas a este tipo de variables en contextos post CAI consideren un muestreo de mayor amplitud y de tipo probabilístico. Tercero, dado que no se contó con datos previos para evaluar procesos de crecimiento posttraumático (Vásquez \& Páez, 2010), sería importante considerar a futuro realizar estudios de tipo longitudinal que permitan verificar algunas ideas planteadas a la luz de los resultados obtenidos previamente. También, sería conveniente complementar los resultados obtenidos con información que entregue la investigación cualitativa, principalmente en torno a los estudios sobre memoria que ya se han realizado en otros contextos (Jelin, 2001).

Finalmente, el estudio de los valores individuales, principalmente a partir de los resultados obtenidos, brinda luces respecto de la importancia de lo relacional y su incidencia en el bienestar de las personas, por lo 
que valores como la cooperación y la asociación servirían como estrategias a fortalecer en futuras intervenciones que busquen una convivencia adecuada (Arango Cálad 2001; Cueto et al., 2016) y una cultura de paz (de Rivera, 2011).

\section{Referencias}

Arango Cálad, C. A. (2001). Hacia una psicología de la convivencia. Revista Colombiana de Psicología, 10, 79-89. Extraído de http://bit.ly/2ledFGI

Arellano Martorellet, J. C. (2011). Identidad social y bienestar en una comunidad rural de la costa norte del Perú (Tesis de Grado, Pontificia Universidad Católica del Perú, Lima, Perú). Extraído de http://tesis.pucp.edu.pe/repositorio/handle/123456789/1175

Argibay, J. C. (2009). Muestra en investigación cuantitativa. Subjetividad y Procesos Cognitivos, 13, 13-29. Extraído de http://www.scielo.org.ar/scielo.php?pid=S1852-73102009000100001\&script=sci_arttext

Balbuena Blengeri, A. C. (2013). Sentido de comunidad, bienestar y memoria colectiva en una comunidad rural de la costa norte del Perú (Tesis de Grado, Pontificia Universidad Católica del Perú, Lima, Perú). Extraído de http://tesis.pucp.edu.pe/repositorio/handle/123456789/1693

Barrenechea, R. (2010). Políticas locales de reparación en Ayacucho iReparaciones sin reparadores? (Documento de Trabajo $\left.\mathrm{N}^{\circ} 157\right)$. Lima, Perú: Instituto de Estudios Peruanos.

Basabe, N., Valencia, J. \& Bobowik, M. (2011). Valores y actitudes: cultura de violencia y paz. En D. Páez, C. M. Beristain, J. L. González, N. Basabe \& J. de Rivera (Eds.), Superando la violencia colectiva y construyendo cultura de paz (pp. 69-101). Madrid, España: Fundamentos.

Batista-Foguet, J. M. \& Coenders, G. (2000). Modelos de ecuaciones estructurales: modelos para el análisis de relaciones causales. Madrid, España: La Muralla.

Becchetti, L., Bruni, L. \& Zamagni, S. (2015). Human values, civil economy, and subjective well-being. En J. Helliwell, R. Layard \& J. Sachs (Eds.), World happiness report 2015 (pp. 132-151). New York, NY: Sustainable Development Solutions Network. Extraído de http://bit.ly/2ldoOaG

Beristain, C. M., Páez, D., Rimé, B. \& Kanyangara, P. (2010). Efectos psicosociales de la participación en rituales de justicia transicional. Revista de Psicología de la Pontificia Universidad Católica del Perú, 28, 9-35. Extraído de http://bit.ly/2ld2r5d

Bilali, R. (2011). Collective memories of intergroup conflict. En D. J. Christie (Ed.), The encyclopedia of peace psychology (Vol. 1). Hoboken, NJ: John Wiley \& Sons. https://doi.org/10.1002/9780470672532.wbepp037

Bilbao, M. Á, Techio, E. M. \& Páez, D. (2007). Felicidad, cultura y valores personales: estado de la cuestión y síntesis meta-analítica. Revista de Psicología de la Pontificia Universidad Católica del Perú, 25, 233-276. Extraído de http://bit.ly/2ldekbE

Bilbao, M. Á., Techio, E., Zubieta, E., Cárdenas, M., Páez, D., Díaz, D. ... Blanco, A. (2011). Bienestar subjetivo y psicológico-social: El impacto de la violencia colectiva. En D. Páez, C. M. Beristain, J. L. González, N. Basabe \& J. de Rivera (Eds.), Superando la violencia colectiva y construyendo cultura de paz (pp. 205-245). Madrid, España: Fundamentos.

Bilsky, W. \& Schwartz, S. H. (1994). Values and personality. European Journal of Personality, 8, 163-181. https://doi.org/10.1002/per.2410080303

Blanco, A. \& Díaz, D. (2005). El bienestar social: su concepto y medición. Psicothema, 17, 582-589. Extraído de http://www.redalyc.org/articulo.oa?id=72717407

Bobowik, M., Basabe, N., Páez, D., Jiménez, A. \& Bilbao, M. A. (2011). Personal values and well-being among Europeans, Spanish natives and immigrants to Spain: Does the culture matter? Journal of Happiness Studies, 12, 401-419. https://doi.org/10.1007/s10902-010-9202-1

Calderón Prada, A., Espinosa Pezzia, A. \& Techio, E. M. (2004). Resiliencia, afrontamiento, bienestar psicológico y clima socio-emocional después de los atentados del 11 de marzo. Ansiedad y Estrés, 10, 265-276.

Cárdenas, M., Páez, D., Rimé, B., Bilbao, A. \& Asún, D. (2014). Personal emotions, emotional climate, social sharing, beliefs, and values among people affected and unaffected by past political violence. Peace and Conflict: Journal of Peace Psychology, 20, 452-464. https://doi.org/10.1037/pac0000038

Cilliers, J., Dube, O. \& Siddiqi, B. (2016). Reconciling after civil conflict increases social capital but decreases individual well-being. Science, 352, 787-794. https://doi.org/10.1126/science.aad9682

Cohen, J. (1988). Statistical power analysis for the behavioral sciences (2a ed.). Hillsdale, NJ: Lawrence Erlbaum.

Comas-Díaz, L., Lykes, M. B. \& Alarcón, R. D. (1998). Ethnic conflict and the psychology of liberation in Guatemala, Peru and Puerto Rico. American Psychologist, 53, 778-792. https://doi.org/10.1037/0003-066X.53.7.778

Comisión de la Verdad y Reconciliación (2003). Informe final. Lima, Perú: Autor.

Conejero, S. \& Etxebarria, I. (2007). The impact of the Madrid bombing on personal emotions, emotional atmosphere and emotional climate. Journal of Social Issues, 63, 273-287. https://doi.org/10.1111/j.1540-4560.2007.00508.x

Cuadra L. H. \& Florenzano U. R. (2003). El bienestar subjetivo: hacia una psicología positiva. Revista de Psicología de la Universidad de Chile, 12(1), 83-96. https://doi.org/10.5354/0719-0581.2003.17380

Cueto, R. M., Espinosa, A., Guillén, H. \& Seminario, M. (2016). Sentido de comunidad como fuente de bienestar en poblaciones socialmente vulnerables de Lima, Perú. Psykhe, 25(1), 1-18. https://doi.org/10.7764/psykhe.25.1.814

Darghouth, S., Pedersen, D., Bibeau, G. \& Rousseau, C. (2006). Painful languages of the body: Experiences of headache among women in two Peruvian communities. Culture, Medicine and Psychiatry, 30, 271-297. https://doi.org/10.1007/s11013-006-9021-3

Defensoría del Pueblo (2013). A diez años de verdad, justicia y reparación. Avances, retrocesos y desafíos de un proceso inconcluso. Lima, Perú: Autor. Extraído de http://bit.ly/2ld9f2Y

de Rivera, J., Kurrien, R. \& Olsen, N. (2007). The emotional climate of nations and their culture of peace. Journal of Social Issues, 63, 255-271. https://doi.org/10.1111/j.1540-4560.2007.00507.x

de Rivera, J. \& Páez, D. (2007). Emotional climate, human security, and cultures of peace. Journal of Social Issues, 63, $233-253$. https://doi.org/10.1111/j.1540-4560.2007.00506.x

de Rivera, J. \& Yurtsever, G. (2010). National emotional climate and the value of freedom. Beliefs and Values, 2, 57-65. https://doi.org/10.1891/1942-0617.2.1.57

de Rivera, J. (2011). La enseñanza de la cultura de paz como un enfoque de la educación para la paz. En D. Páez, C. M. Beristain, J. L. González, N. Basabe \& J. de Rivera (Eds.), Superando la violencia colectiva y construyendo cultura de paz (pp. 569-585). Madrid, España: Fundamentos. 
Diener, E., Emmons, R. A., Larsen, R. J. \& Griffin, S. (1985). The Satisfaction With Life Scale. Journal of Personality Assessment, 49, 71-75. https://doi.org/10.1207/s15327752jpa4901_13

Diener, E. \& Ryan, K. (2009). Subjective well-being: A general overview. South African Journal of Psychology, 39, $391-406$. https://doi.org/10.1177/008124630903900402

Espinosa, A., Schmitz, M. \& Cueto, R. M. (2015). Impacto psicosocial de la Comisión de la Verdad y Reconciliación (CVR) en una muestra de estudiantes universitarios de Lima-Perú. Psicología Política, 15, 157-184. Extraído de http://pepsic.bvsalud.org/pdf/rpp/v15n32/v15n32a11.pdf

Espinosa, A. \& Tapia, G. (2011). Identidad nacional como fuente de bienestar subjetivo y social. Boletín de Psicología, 102, 71-87. Extraído de http://bit.ly/2ljLJhp

Ferrándiz, J. (2011). Identidad social y clima socio-emocional en una comunidad rural de la costa norte del Perú (Tesis de Grado, Pontificia Universidad Católica del Perú, Lima, Perú). Extraído de http://tesis.pucp.edu.pe:8080/repositorio/handle/123456789/690?show=full

Gargurevich, R. \& Matos, L. (2012). Validez y confiabilidad de Escala de Afecto Positivo y Negativo (SPANAS) en estudiantes universitarios peruanos. Revista de Psicología de la Universidad César Vallejo, 14(2), 208-217. Extraído de http://revistas.ucv.edu.pe/index.php/R_PSI/article/view/196/105

Gouveia, V. V. (2003). A natureza motivacional dos valores humanos: evidências acerca de uma nova tipologia [La naturaleza motivacional de los valores humanos: evidencias sobre una nueva tipología]. Estudos de Psicologia (Natal), 8, 431-443. https://doi.org/10.1590/S1413-294X2003000300010

Gouveia, V. V., Milfont, T. L. \& Guerra, V. M. (2014a). Functional theory of human values: Testing its content and structure hypotheses. Personality and Individual Differences, 60, 41-47. https://doi.org/10.1016/j.paid.2013.12.012

Gouveia, V. V., Milfont, T. L. \& Guerra, V. M. (2014b). The functional theory of human values: From intentional overlook to first acknowledgementA reply to Schwartz (2014). Personality and Individual Differences, 68, 250-253. https://doi.org/10.1016/j.paid.2014.03.025

Gouveia, V. V., Santos, W. S., Milfont, T. L., Fischer, R., Clemente, M. \& Espinosa, P. (2010). Teoría funcionalista de los valores humanos en España: comprobación de las hipótesis de contenido y estructura. Revista Interamericana de Psicología, 44, 213-224. Extraído de http://bit.ly/2ljpEzv

Gouveia, V. V., Vione, K. C., Milfont, T. L. \& Fischer, R. (2015). Patterns of value change during the life span: Some evidence from a functional approach to values. Personality and Social Psychology Bulletin, 41, 1276-1290. https://doi.org/10.1177/0146167215594189

Halloran, M. J. (2007). Indigenous reconciliation in Australia: Do values, identity and collective guilt matter? Journal of Community \& Applied Social Psychology, 17, 1-18. https://doi.org/10.1002/casp.876

Hernández Sampieri, R., Fernández Collado, C. \& Baptista Lucio, M. P. (2010). Metodología de la investigación (5a ed.). México DF, México: McGraw Hill.

Holtz, P. \& Wagner, W. (2011). Dehumanization, infrahumanization, and naturalization. En D. J. Christie (Ed.), The encyclopedia of peace psychology (Vol. 1). Hoboken, NJ: John Wiley \& Sons. https://doi.org/10.1002/9780470672532.wbepp079

Inglehart, R. (1977). The silent revolution: Changing values and political styles among western publics. Princeton, NJ: Princeton University Press.

Instituto de Defensa Legal (2015). Seguridad ciudadana: informe anual 2015. Lima, Perú: Autor.

Instituto de Estudios Peruanos (2016). Baja confianza en las instituciones peruanas afecta el ejercicio de su autoridad. Lima, Perú: Autor.

Instituto Nacional de Estadística e Informática (2016). Estadísticas de seguridad ciudadana: abril-setiembre 2016 (Informe Técnico N ${ }^{\circ}$ 4). Lima, Perú: Autor.

Jamaludin, N. L., Sam, D. L., Sandal, G. M. \& Adam, A. A. (2016). Personal values, subjective well-being and destination-loyalty intention of international students. Springer-Plus, 5, Article 720. https://doi.org/10.1186/s40064-016-2439-3

Jelin, E. (2001). Los trabajos de la memoria. Madrid, España: Siglo XXI.

Kendall, R., Matos, L. J. \& Cabra, M. (2006). Salud mental en el Perú, luego de la violencia política. Intervenciones itinerantes. Anales de la Facultad de Medicina de la Universidad Nacional Mayor de San Marcos, 67, 184-190. https://doi.org/10.15381/anales.v67i2.1258

Keyes, C. L. M. (1998). Social well-being. Social Psychology Quarterly, 61, 121-140. https://doi.org/10.2307/2787065

Kline, R. B. (2005). Principles and practice of structural equation modeling (2a ed.). New York, NY: Guilford Press.

Larisgoitia, I., Izarzugaza, I., Markez, I., Fernández, I., Iraurgi, I., Ballesteros, J. ... Retolaza, A. (2009). La noche de las víctimas: investigación sobre el impacto en la salud de la violencia colectiva (ISAVIC) en el País Vasco. Bilbao, España: Fundazioa Fernando Buesa Blanco.

León Urteaga, E. A. (2012). Percepción del contexto nacional y su relación con el clima emocional, el bienestar y la identidad nacional en una muestra de estudiantes de Cajamarca, Lima y Pucallpa (Tesis de Grado, Pontificia Universidad Católica del Perú, Lima, Perú). Extraído de http://tesis.pucp.edu.pe/repositorio/handle/123456789/1698

Levitt, J. M. (2011). Post-traumatic stress disorder and peace. En D. J. Christie (Ed.), The encyclopedia of peace psychology (Vol. 3). Hoboken, NJ: John Wiley \& Sons. https://doi.org/10.1002/9780470672532.wbepp214

Lykes, M. B., Beristain, C. M. \& Cabrera Pérez-Armiñan, M. L. (2007). Political violence, impunity, and emotional climate in Maya communities. Journal of Social Issues, 63, 369-385. https://doi.org/10.1111/j.1540-4560.2007.00514.x

Martínez Martínez, M. C., Paterna Bleda, C. \& Gouveia, V. V. (2006). Relevancia del modelo dual de valores en relación con el prejuicio y la intención de contacto hacia exogrupos. Anales de Psicología, 22, 243-250. Extraído de http://bit.ly/2lcYCNt

Mezulis, A. H., Abramson, L. Y., Hyde, J. S. \& Hankin, B. L. (2004). Is there a universal positivity bias in attributions? A meta-analytic review of individual, developmental, and cultural differences in the self-serving attributional bias. Psychological Bulletin, 130, 711747. https://doi.org/10.1037/0033-2909.130.5.711

Oishi, S., Diener, E. F., Lucas, R. E. \& Suh, E. M. (1999). Cross-cultural variations in predictors of life satisfaction: Perspectives from needs and values. Personality and Social Psychology Bulletin, 25, 980-990. https://doi.org/10.1177/01461672992511006

Ordinola Macha, M. M. (2012). Satisfacción con la vida, clima emocional y tendencia al conflicto en pobladores de Cajamarca (Tesis de Grado, Pontificia Universidad Católica del Perú, Lima, Perú). Extraído de http://tesis.pucp.edu.pe/repositorio/handle/123456789/1442

Organización Mundial de la Salud (2002). Informe mundial sobre la violencia y la salud: resumen. Washington, DC: Organización Panamericana de la Salud.

Páez, D., Fernández, I. \& Beristain, C. M. (2001). Catástrofes, traumas y conductas colectivas: procesos y efectos culturales. En C. San Juan (Coord.), Catástrofes y ayuda de emergencia: estrategias de evaluación, prevención y tratamiento (pp. 85-148). Barcelona, España: Icaria.

Páez, D., Ruiz, J. I., Gailly, O., Kornblit, A. L., Wiesenfeld, E. \& Vidal, C. M. (1997). Clima emocional: su concepto y medición mediante una investigación transcultural. Revista de Psicología Social, 12, 79-98. https://doi.org/10.1174/021347497320892045 
Puyana, A. (2015). Desigualdad horizontal y discriminación étnica en cuatro países latinoamericanos: notas analiticas para una propuesta de políticas (Series de la CEPAL LC/L.3973). México DF, México: Comisión Económica para América Latina y el Caribe.

Rokeach, M. (1973). The nature of human values. New York, NY: Free Press.

Ruiz, M. A., Pardo, A. \& San Martín, R. (2010). Modelos de ecuaciones estructurales. Papeles del Psicólogo, 31, 34-45. Extraído de http://bit.ly/2le0MMP

Ryan, R. M. \& Deci, E. L. (2001). On happiness and human potentials: A review of research on hedonic and eudaimonic well-being. Annual Review of Psychology, 52, 141-166. https://doi.org/10.1146/annurev.psych.52.1.141

Sagiv, L. \& Schwartz, S. H. (2000). Value priorities and subjective well-being: Direct relations and congruity effects. European Journal of Social Psychology, 30, 177-198. https://doi.org/10.1002/(SICI)1099-0992(200003/04)30:2\%3C177::AID-EJSP982\%3E3.0.CO;2-Z

Schwartz, S. H. (1994). Are there universal aspects in the structure and contents of human values? Journal of Social Issues, 50(4), 1945. https://doi.org/10.1111/j.1540-4560.1994.tb01196.x

Schwartz, S. H. (2005). Robustness and fruitfulness of a theory of universals in individual human values. En A. Tamayo \& J. B. Porto (Orgs.), Valores e trabalho [Valores y trabajo] (pp. 56-95). Petrópolis, Brasil: Vozes.

Schwartz, S. H. (2014). Functional theories of human values: Comment on Gouveia, Milfont, and Guerra (2014). Personality and Individual Differences, 68, 247-249. https://doi.org/10.1016/j.paid.2014.03.024

Schwartz, S. H., Cieciuch, J., Vecchione, M., Davidov, E., Fischer, R., Beierlein, C. ... Konty, M. (2012). Refining the theory of basic individual values. Journal of Personality and Social Psychology, 103, 663-688. https://doi.org/10.1037/a0029393

Seminario Obando, M. A. N. (2014). Sentido de comunidad, participación comunitaria y valores en líderes/as comunitarios/as en contextos de vulnerabilidad social (Tesis de Grado, Pontificia Universidad Católica del Perú, Lima, Perú). Extraído de http://tesis.pucp.edu.pe/repositorio/handle/123456789/5819

Sheldon, K. M., Elliot, A. J., Ryan, R. M., Chirkov, V., Kim, Y., Wu, C. ... Sun, Z. (2004). Self-concordance and subjective well-being in four cultures. Journal of Cross-Cultural Psychology, 35, 209-223. https://doi.org/10.1177/0022022103262245

Sheldon, K. M. \& Kasser, T. (1995). Coherence and congruence: Two aspects of personality integration. Journal of Personality and Social Psychology, 68, 531-543. https://doi.org/10.1037/0022-3514.68.3.531

Shmotkin, D. \& Lomranz, J. (1998). Subjective well-being among Holocaust survivors: An examination of overlooked differentiations. Journal of Personality and Social Psychology, 75, 141-155. https://doi.org/10.1037/0022-3514.75.1.141

Snider, L., Cabrejos, C., Huayllasco Marquina, E., Trujillo, J. J., Avery, A. \& Ango Aguilar, H. (2004). Psychosocial assessment for victims of violence in Peru: The importance of local participation. Journal of Biosocial Science, 36, 389-400. https://doi.org/10.1017/S0021932004006601

Sortheix, F. M. \& Lönnqvist, J. -E. (2015). Person-group value congruence and subjective well-being in students from Argentina, Bulgaria and Finland: The role of interpersonal relationships. Journal of Community \& Applied Social Psychology, 25, 34-48. https://doi.org/10.1002/casp.2193

Tay, L. \& Diener, E. (2011). Needs and subjective well-being around the world. Journal of Personality and Social Psychology, 101, 354365. https://doi.org/10.1037/a0023779

Techio, E., Zubieta, E., Páez, D., de Rivera, J., Rimé, B. \& Kanyangara, P. (2011). Clima emocional y violencia colectiva: el estado de la cuestión e instrumentos de medición. En D. Páez, C. M. Beristain, J. L. González, N. Basabe \& J. de Rivera (Eds.), Superando la violencia colectiva y construyendo cultura de paz (pp. 103-148). Madrid, España: Fundamentos.

Theidon, K. (1999). Domesticando la violencia: el alcohol y las secuelas de la guerra. Ideele, 120, 56-62.

Thorp, R. (2010). Desigualdad, etnicidad y conflicto: entendiendo las interrelaciones [Entrevistada por Carlos de los Ríos]. Argumentos: Revista de Análisis y Crítica, 2, 55-62. Extraído de http://revistaargumentos.iep.org.pe/wp-content/uploads/2014/01/A\%C3\%B1o-4No.-2-Mayo-2010.pdf

Vázquez, C. \& Páez, D. (2010). Posttraumatic growth in Spain. En T. Weiss \& R. Berger (Eds.), Posttraumatic growth and culturally competent practice: Lessons learned from around the globe (pp. 97-112). Hoboken, NJ: John Wiley and Sons.

Watson, D., Clark, L. A. \& Tellegen, A. (1988). Development and validation of brief measures of positive and negative affect: The PANAS scales. Journal of Personality and Social Psychology, 54, 1063-1070. https://doi.org/10.1037/0022-3514.54.6.1063

Wessells, M. \& Kostelny, K. (2011). Psychosocial impact of political violence. En D. J. Christie (Ed.), The encyclopedia of peace psychology (Vol. 3). Hoboken, NJ: John Wiley and Sons. https://doi.org/10.1002/9780470672532.wbepp226

Zubieta, E. M., Muratori, M. \& Mele, S. (2012). Bienestar, clima emocional, percepción de problemas sociales y confianza. Anuario de Investigaciones de la Universidad de Buenos Aires, 19, 97-106. Extraído de http://bit.ly/2ld6r66

Fecha de recepción: Enero de 2016.

Fecha de aceptación: Septiembre de 2017. 